\title{
The autoclavable semirigid thoracoscope: the way forward in pleural disease?
}

\author{
M. Munavvar*, M.A.I. Khan*, J. Edwards* , Z. Waqaruddin* and J. Mills
}

ABSTRACT: Medical thoracoscopy is a valuable tool in the investigation and management of pleural disease. It has considerable advantages over conventional blind pleural biopsy and videoassisted thoracoscopic surgery. Despite this, the practice of this technique in the UK is limited. Most operators use the rigid thoracoscope, which may be an unfamiliar instrument to respiratory physicians. A semirigid thoracoscope is available but its use has not been possible in the UK as it requires sterilisation with ethylene oxide, which is not approved in this country. The present authors describe herein their experience with the first ever autoclavable semirigid thoracoscope.

Medical thoracoscopy using the new instrument was performed in $\mathbf{5 6}$ patients between June 2004 and May 2006. All patients had been referred with a unilateral pleural effusion of unknown aetiology, where blind pleural aspiration had failed to yield an answer. Diagnostic samples were taken and talc poudrage performed where appropriate.

The instrument was easy to handle and excellent views were obtained. Histologically adequate biopsy samples were obtained in $\mathbf{5 4}$ patients. The combination of clinical features, computed tomography findings and thoracoscopic biopsy enabled a definite diagnosis in 49 (90.7\%) of these patients. There were no complications.

The autoclavable semirigid thoracoscope has immense potential in the diagnosis and management of pleural disease. Its diagnostic yield in pleural disease is comparable to the conventional rigid thoracoscope. It is similar in design to the fibreoptic bronchoscope and respiratory physicians should be able to adapt to its use easily. It is also compatible with existing video processors and light sources available in most endoscopy suites. The fact that this instrument is autoclavable should open the field for its use in the UK as well as in other countries.

KEYWORDS: Malignancy, pleural biopsy, pleurodesis, semirigid, thoracoscopy

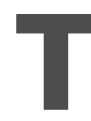

he accurate diagnosis of pleural disease can present a considerable challenge. After thoracocentesis and/or blind pleural biopsy, $\sim 25-40 \%$ of pleural abnormalities remain undiagnosed [1, 2]. Medical thoracoscopy refers to the examination of the pleural space in a nonintubated patient under conscious sedation [3]. It enables inspection of the pleural surfaces, the taking of pleural biopsies under direct vision, therapeutic drainage of effusions and pleurodesis in one sitting. It enables a positive diagnosis in $>90 \%$ of pleural effusions $[4,5]$. It is very well tolerated and there is no requirement for a general anaesthetic, as opposed to video-assisted thoracoscopic surgery (VATS).

Despite its obvious benefits, the practice of thoracoscopy in the UK, and indeed in most parts of the world, is not widespread [6, 7]. A reason suggested for this is that most respiratory physicians are not familiar with the use of the more commonly used rigid thoracoscope [8]. The procedure has been attempted with the flexible bronchoscope but this is difficult to manipulate within the pleural cavity and results have been inferior to the rigid instrument $[9,10]$.

The semirigid thoracoscope was developed in an attempt to combine the best features of the flexible and rigid instruments [3]. However, the use of this instrument in the UK has not been possible as it requires sterilisation with ethylene oxide, the use of which is not allowed in the UK.

The present authors describe their experience with the first ever autoclavable semirigid thoracoscope. This instrument is compatible with the video processors and light sources available in most bronchoscopy units.

\section{MATERIALS AND METHODS}

The present study was a single-centre study and included consecutive patients referred over an 18-month period with a unilateral pleural effusion and a negative or unsuccessful blind pleural
AFFILIATIONS

Depts of *Respiratory Medicine,

${ }^{\text {\#} H i s t o p a t h o l o g y, ~ a n d ~}$

"Chris Chell Endoscopy Unit, Royal

Preston Hospital, Preston, UK.

CORRESPONDENCE

M. Munavvar

Dept of Respiratory Medicine

Lancashire Teaching Hospitals

NHS Trust

Royal Preston Hospital

Fulwood

Preston PR2 9HT

UK

Fax: 441772522410

E-mail: Mohammed.Munavvar@

Ithtr.nhs.uk

Received:

August 032006

Accepted after revision:

December 192006

SUPPORT STATEMENT

Olympus KeyMed UK (Southend-on-

Sea, UK) provided the semirigid

thoracoscope for this study.

STATEMENT OF INTEREST

None declared.

European Respiratory Journa

Print ISSN 0903-1936

Online ISSN 1399-3003 
fluid aspirate. All patients had a contrast computed tomography (CT) scan of the thorax before entry. Patients with "highly suspicious" pleural fluid cytology were not entered into the study unless the cytological diagnosis was at variance with clinical features and CT findings. All patients with merely "suspicious" or "normal" cytology were included in the study group.

The instrument employed was a prototype semirigid thoracoscope (LTF-160Y1; Olympus, Tokyo, Japan; supplied by Olympus KeyMed UK, Southend-on-Sea, UK). It has controls similar to that of a flexible fibreoptic bronchoscope. The total length of the instrument is $52 \mathrm{~cm}$, with the insertion portion being $27 \mathrm{~cm}$ long. Of this, the proximal $22 \mathrm{~cm}$ are rigid and the distal $5 \mathrm{~cm}$ are flexible. The external diameter of the insertion portion is $7 \mathrm{~mm}$. The tip is bendable in one plane, with an upward angulation of $160^{\circ}$ and downward angulation of $130^{\circ}$ (fig. 1). The 2.8-mm inner working channel accommodates the biopsy forceps and other instruments. It has an yttriumaluminium-garnet $810-\mathrm{nm}$ diode laser and high-frequency compatibility. It is compatible with the EVIS EXERA 160 and 145 and EVIS 100 and 140 Series video processors and light sources (Olympus). Sterilisation is achieved by autoclaving.
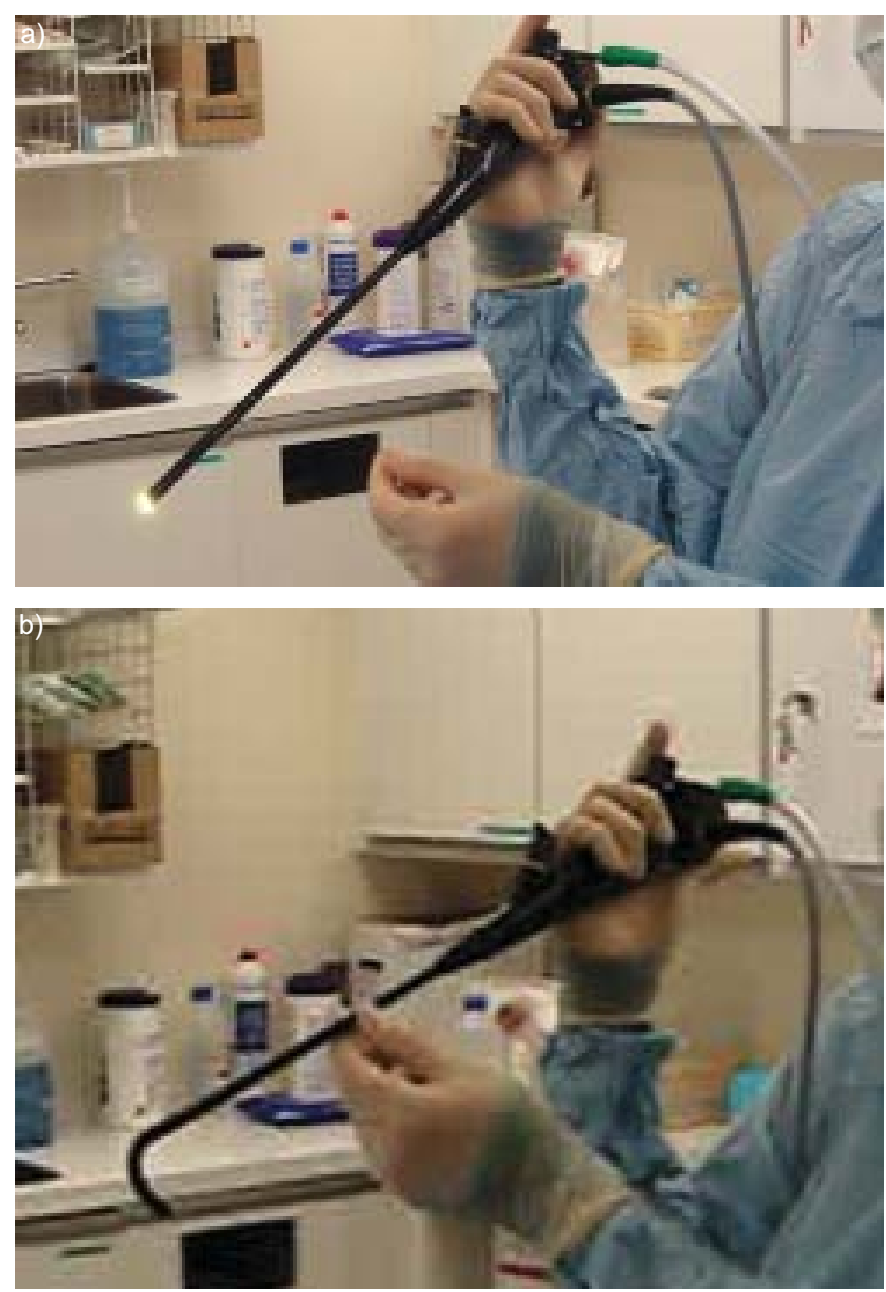

FIGURE 1. The instrument (a) has a flexible tip (b), as shown.

\section{Procedure}

All procedures were performed by a single operator (M. Munavvar) in the endoscopy suite of the present authors' institution (Royal Preston Hospital, Preston, UK). Informed consent was obtained in writing. A single puncture technique was used. Full surgical aseptic technique was adhered to. The lateral decubitus position was employed, with the diseased side up. All patients received combination sedation with intravenous midazolam and alfentanil; oxygen saturations and heart rate were monitored throughout. Patients received supplementary oxygen by nasal cannulae routinely.

Lignocaine was employed for local anaesthesia. The presence of fluid was first confirmed by aspiration; if this failed, the patient's position was changed and aspiration was attempted again. After fluid was obtained, an incision was placed in the midaxillary line and a $10-\mathrm{mm}$ trocar inserted. The thoracoscope was then inserted and, following drainage of all fluid to dryness, the pleural surfaces were inspected. Pleural fluid and parietal pleural biopsy samples were obtained where indicated. Between six and 10 biopsies were taken per patient. An FB-240K oval fenestrated biopsy forceps (Olympus) was used. Talc poudrage with $5 \mathrm{~g}$ sterile talc (Novatech, La Ciotat, France) was carried out where appropriate. A 24F chest drain (Portex; Smiths Medical International Ltd, Hythe, UK) was inserted through the trocar. All patients underwent a chest radiograph after the procedure. The chest tube was removed as soon as full expansion of the lung was confirmed radiologically.

The operator recorded the image quality, the presence of pleural abnormalities on inspection, the duration of chest drainage after the procedure and the occurrence of complications.

\section{RESULTS}

Between June 2004 and May 2006, 60 procedures were attempted on 59 patients. Three procedures were abandoned before insertion of the trocar as no fluid was obtained on initial aspiration despite multiple attempts. Hence, 57 procedures were performed on 56 patients. The indication in all the procedures was a unilateral pleural effusion of unknown cause, with no diagnosis on blind pleural fluid aspiration. The median age of the patients was 68.5 yrs. The range of ages was $20-90$ yrs, with 19 patients aged $\geqslant 75$ yrs. There were 37 males and 19 females.

The thoracoscope was found to be easy to handle. The image quality was uniformly excellent (fig. 2) and fluid was suctioned without difficulty. In two patients, extensive adhesions precluded satisfactory inspection and hence adequate biopsies could not be taken. Several other patients had moderate adhesions but the flexible tip was easily manoeuvred round them, enabling inspection and biopsy. Adequate inspection of the pleural cavity was undertaken in 54 patients; in all but one of these, histologically satisfactory biopsy samples were obtained. This patient, who was only the third to be examined, underwent a repeat procedure at which a satisfactory sample was taken.

In the end, satisfactory biopsy samples were obtained in 54 patients. The combination of clinical features, contrast CT scanning and thoracoscopic inspection/biopsy enabled a 

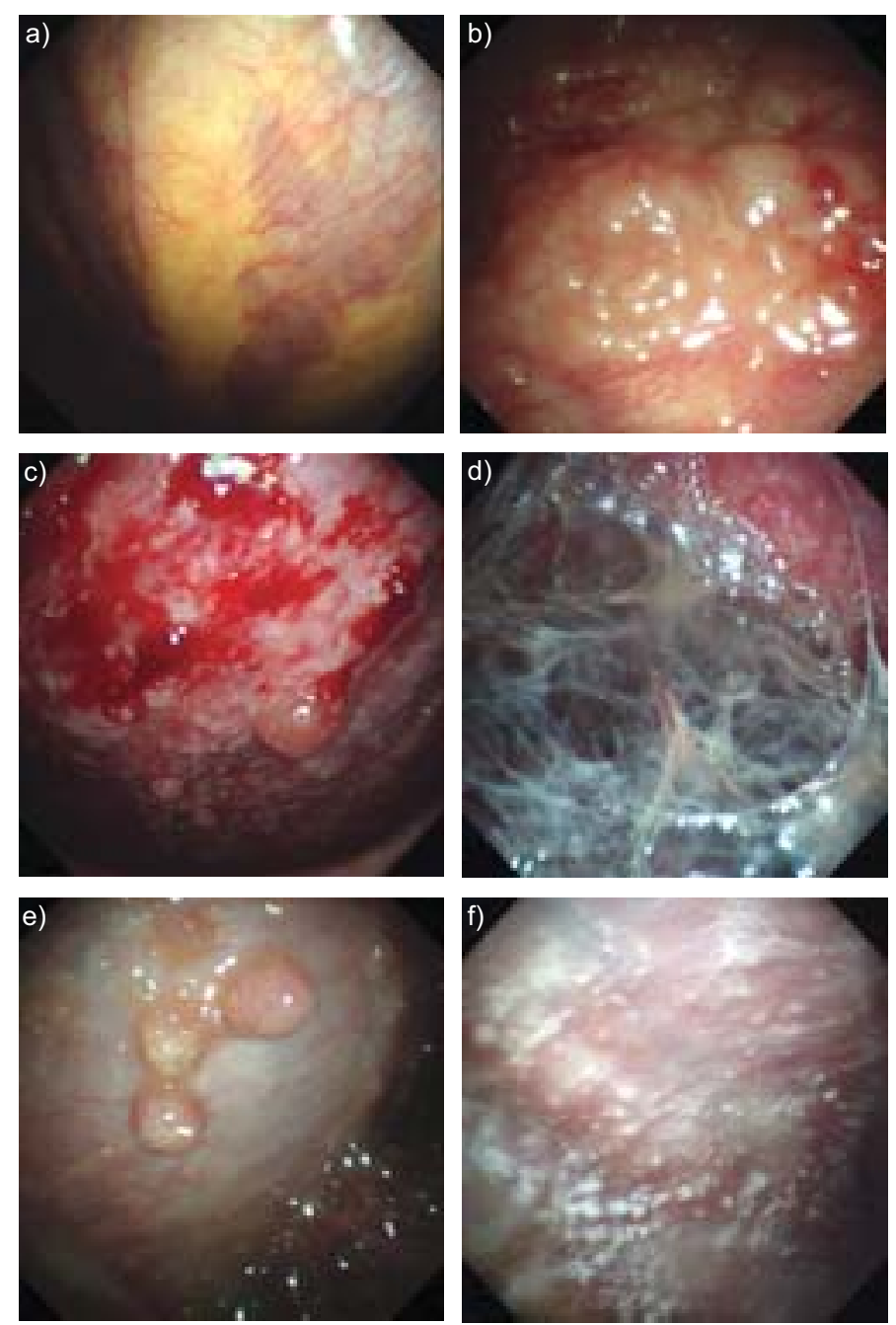

FIGURE 2. Images taken using the thoracoscope showing a) normal pleura b) tuberculosis, c) mesothelioma, d) mesothelioma with adhesions, e) adenocarcinoma and f) talc poudrage.

definite diagnosis in 49 of these, giving a positive yield of $90.7 \%$ (table 1 ).

In the two patients found to have empyema, this diagnosis had not been suspected before thoracoscopy and was only established after purulent fluid was obtained with the instrument. In all the patients with a nonmalignant diagnosis on histology, there was no reason to suspect malignancy on clinical or CT grounds, and hence no indication for further investigation with thoracotomy.

The patients with histological diagnoses of inflammation, fibrosis and normal pleura had a prior history suggestive either of infection or of asbestos exposure, except one who was known to have rheumatoid arthritis. The final clinical diagnoses in these patients were therefore post-pneumonic effusion, benign asbestos-related pleural effusion and rheumatoid effusion. The subsequent clinical course of these patients has given no cause to believe otherwise.

For the five patients where no diagnosis was obtained from thoracoscopy despite satisfactory sampling, the following

\begin{tabular}{ll} 
TABLE 1 & $\begin{array}{l}\text { Final diagnosis in patients undergoing } \\
\text { thoracoscopy with adequate sampling }\end{array}$ \\
& Frequency \\
Diagnosis & 15 \\
\hline & 8 \\
Mesothelioma & 7 \\
Bronchogenic carcinoma & 3 \\
Metastatic carcinoma & 1 \\
Tuberculosis & 1 \\
Non-Hodgkin's lymphoma & 2 \\
Sarcomatoid carcinoma of pleura & 7 \\
Empyema & 3 \\
Inflammation & 1 \\
Fibrosis/fibrin & 1 \\
Pleural plaque & 5 \\
Normal pleura & \\
No diagnosis from thoracoscopy &
\end{tabular}

events occurred. 1) The first patient had considerable adhesions. Metastatic adenocystic salivary gland carcinoma was ultimately diagnosed on ultrasound-guided pleural biopsy. 2) In the second patient, there was a prior history of colorectal carcinoma. Metastatic colorectal carcinoma was ultimately diagnosed by gastro-intestinal and gynaecological investigations. 3) The third patient was known to have had gastric non-Hodgkin's lymphoma in the past. With no answer forthcoming from thoracoscopy, a clinical decision to treat for relapsed lymphoma was made. With treatment, the patient's pleural disease completely resolved. 4) One patient was diagnosed with nonsmall cell lung cancer by transbronchial needle aspiration. 5) The remaining patient proceeded to a VATS biopsy, which was also negative. The patient eventually underwent a thoracotomy. Adenocarcinoma of the lung was diagnosed.

All patients tolerated the procedure well. Pharmacological reversal of sedation was not required in any cases. There were no complications and no mortality. The chest drain was removed within $24 \mathrm{~h}$ in the majority and $48 \mathrm{~h}$ in all patients.

\section{DISCUSSION}

When faced with the need to obtain a pleural biopsy specimen, many UK physicians resort to a blind procedure with the Cope or Abrams needle. Blind pleural biopsy has a notoriously poor yield and patients in whom no result is obtained are often referred for a VATS procedure [1, 2]. This is expensive and requires general anaesthesia with single lung ventilation and the use of an operating theatre. In the UK, access to VATS is limited due to the relatively small number of surgeons who practise it and often patients have to wait an unacceptably long period of time for a procedure. In addition, patients with advanced pleural disease are often frail with multiple comorbidities and may not be suitable for a general anaesthetic in the first place.

The British Thoracic Society (BTS) recommends either thoracoscopy or image-guided biopsy (using CT or ultrasound) as the next line of investigation in the event of a nondiagnostic blind pleural aspirate [11]. CT-guided biopsy is safe, with the only reported complications being local haematoma and minor haemoptysis. It was found to be quite sensitive $(87 \%)$ in a 
series of 50 patients [12]. Unfortunately, it does not afford the opportunity for drainage of pleural fluid or pleurodesis in the same sitting. Indeed, the BTS recommends thoracoscopy in the event of image-guided biopsy not yielding an answer.

Medical thoracoscopy is extremely safe, with major and minor complication rates of 1.9 and $5.6 \%$, respectively, in one series [13]. Death as a complication is extremely rare, with only one fatality out of 8,000 patients in one series [14]. Thoracoscopy also offers the opportunity to perform diagnostic sampling, aspiration of fluid and talc poudrage in the same setting. Inpatient stay is remarkably short.

The practice of medical thoracoscopy in the UK remains limited, for reasons already discussed. Indeed, a 2004 survey showed that only $14 \%$ of UK respiratory physicians had any exposure to it at all, and only $6 \%$ had performed $>10$ procedures [6].

The semirigid instrument used in the present study may offer a way forward. It appears to have some advantages over the rigid thoracoscope. With its similarity in design to the flexible bronchoscope, it is hoped that chest physicians will be able to adapt to its use without too much difficulty, although formal training is essential [8]. It is easy to manoeuvre within the pleural cavity. It is compatible with standard biopsy forceps and can be used with the processors and light sources found in most endoscopy rooms.

Undoubtedly, the biopsy size from the rigid thoracoscope is larger than with the semirigid instrument. This has been quoted as a reason for the former's superiority. However, smaller biopsy size does not necessarily translate to inferior diagnostic yield; indeed, the present authors' results, as well as those of other operators, have been excellent $[3,15]$. The fact that the instrument used can be autoclaved is a huge bonus and it opens the way for its wider use both in the UK and abroad.

Overall, there is immense potential for the use of the autoclavable semirigid thoracoscope in the speedy and accurate diagnosis and effective management of pleural disease.

\section{REFERENCES}

1 Poe RH, Israel RH, Utell MJ, et al. Sensitivity, specificity, and predictive values of closed pleural biopsy. Arch Intern Med 1984; 144: 325-328.
2 Prakash UBS, Relman H. Comparison of needle biopsy with cytologic analysis for the evaluation of pleural effusion: analysis of 414 cases. Mayo Clin Proc 1985; 60: 158-164.

3 Ernst A, Hersh CP, Thurer R, et al. A novel instrument for the evaluation of the pleural space. Chest 2002; 122: 1530-1534.

4 Canto A, Blasco E, Casillas M, et al. Thoracoscopy in the diagnosis of pleural effusion. Thorax 1977; 32: 550-554.

5 Weissberg D, Kaufmann M. Diagnostic and therapeutic pleuroscopy. Experience with 27 patients. Chest 1980; 78: 732-735.

6 Munavvar M, Shakur R, Corless M, Singh H, Guirguis A. Survey of the practice of interventional bronchoscopy in UK. Thorax 2004; 59: Suppl. II, P76.

7 Burrows NJ, Ali NJ, Cox GM. The use and development of medical thoracoscopy in the United Kingdom over the past 5 years. Respir Med 2006; 100: 1234-1238.

8 Mathur PN. Pleuroscopy: a window to the pleura. J Bronchol 2004; 11: 147-149.

9 Oldenburg FA Jr, Newhouse MT. Thoracoscopy. A safe, accurate diagnostic procedure using the rigid thoracoscope and local anesthesia. Chest 1979; 75: 45-50.

10 Davidson AC, George RJ, Sheldon CD, et al. Thoracoscopy: assessment of physician service and comparison of a flexible bronchoscope used as a thoracoscope with a rigid thoracoscope. Thorax 1988; 43: 327-332.

11 Maskell NA, Butland RJA, on behalf of the British Thoracic Society Pleural Disease Group. BTS guidelines for the investigation of a unilateral pleural effusion in adults. Thorax 2003; 58: Suppl. II, ii8-ii17.

12 Maskell NA, Gleeson FV, Davies RJO. Standard pleural biopsy versus CT-guided cutting-needle biopsy for diagnosis of malignant disease in pleural effusions: a randomised controlled trial. Lancet 2003; 361: 1326-1330.

13 Menzies R, Charbonneau M. Thoracoscopy for the diagnosis of pleural disease. Ann Intern Med 1991; 114: 271-276.

14 Boutin C, Viallat JR, Cargnino P, et al. Thoracoscopy in malignant pleural effusion. Am Rev Respir Dis 1981; 124: 588-592.

15 Khan MAI, Ambalavanan S, Munavvar M, et al. A comparison of the diagnostic yield of rigid and semirigid thoracoscopes. Eur Respir J 2006; 28: Suppl. 50, 970s. 